\section{Perfil de utilização de medicamentos em idosos da zona urbana de Santa Rosa, Rio Grande do Sul, Brasil}

\author{
Use of medicines by the elderly in Santa Rosa, \\ Rio Grande do Sul State, Brazil
}

\author{
1 Departamento de Ciências \\ da Saúde, Universidade \\ Regional do Noroeste do \\ Estado do Rio Grande do Sul, \\ Santa Rosa, Brasil. \\ 2 Fundação Municipal de \\ Saúde de Santa Rosa, Santa \\ Rosa, Brasil. \\ Correspondência \\ $V$. B. Flores \\ Departamento de Ciências \\ da Saúde, Universidade \\ Regional do Noroeste do \\ Estado do Rio Grande do Sul. \\ Rua Taquara 644, Santa \\ Rosa, RS \\ 98900-000, Brasil. \\ vafflores@yahoo.com.br
}

\begin{abstract}
Medicines are one of the most important health care items for the elderly, who are particularly prone to use multiple drugs with a higher number of adverse reactions. The current study aimed to assess the profile of pharmaceutical consumption by the elderly. A cross-sectional epidemiological study used household interviews in a random sample of 294 elderly living in Santa Rosa, Rio Grande do Sul State, Brazil. The elderly represent $9 \%$ of the local population. The results showed $a$ mean consumption of 2.79 medicines per individual. The prevalence rate for use of medicines in the previous week was $82 \%$, and of the 827 medicines used by the elderly, 794 (96\%) had been prescribed. The most widely used pharmacological classes were anti-hypertensives (21.3\%) and diuretics (11.3\%). Prevalence of pharmaceutical use was comparable to the results from other studies, thus confirming the importance of medicines in the health of the elderly. The percentage of medicines used without a medical prescription was low. The most frequently reported medicines and formulations were those usually prescribed and dispensed free of cost in the municipal basic health care system, which has been organized for more than 10 years, with coverage of nearly $100 \%$.
\end{abstract}

Pharmaceutical Preparations; Aged; Pharmacoepidemiology
Vanessa Boeira Flores 1,2

Luís Antônio Benvegnú 1,2

\section{Introdução}

O aumento da população idosa no Brasil segue uma tendência já ocorrida em países desenvolvidos, trazendo cada vez mais desafios aos serviços e aos profissionais de saúde. Entre as décadas de 1940 e 1970, a expectativa de vida aumentou devido, sobretudo às ações de saúde pública como vacinação e saneamento básico e aos avanços médico-tecnológicos 1 .

Com o aumento da população idosa serão necessárias mudanças no modelo de atenção à saúde no país, principalmente em relação à assistência farmacêutica prestada à população idosa 2 .

No Brasil, estudos populacionais sobre o consumo de medicamentos evidenciam o uso crescente com a idade, tanto em pequenos povoados do interior 3 como em grandes centros urbanos 4,5. Assim como o número de indivíduos idosos vem aumentando, o consumo de medicamento por esta população acompanha esta tendência. Os idosos são, possivelmente, o grupo etário mais medicalizado na sociedade, devido ao aumento da prevalência de doenças crônicas com a idade 6 .

Assim, os medicamentos representam um dos itens mais importantes da atenção à saúde do idoso. As causas de adoecimento e morte neste grupo específico são de etiologia multifatorial e funcional. Associa-se a isso a imensa variedade e disponibilidade de especialidades farmacêuti- 
cas e associações de fármacos sem racionalidade terapêutica que justifique a sua comercialização no vasto mercado farmacêutico. Observa-se também que os medicamentos são alvos de medidas que estimulam o seu consumo como: propagandas, descontos e promoções, tendo na maioria das vezes o idoso como público alvo 1 .

É comum encontrar, nas prescrições médicas de idosos, dosagens inadequadas, interações medicamentosas, associações e redundância - uso de fármacos pertencentes a uma mesma classe terapêutica - e medicamentos sem valor terapêutico. É possível afirmar que, mais do que em qualquer outro grupo etário, os medicamentos são prescritos para os idosos sem haver clara correspondência entre a doença e a ação farmacológica ${ }^{7}$. Estes são, equivocadamente empregados como conseqüência de uma cultura de medicalização, impulsionada pelo valor simbólico dado aos medicamentos.

Além disso, os idosos apresentam limitações fisiológicas, e com isso a farmacocinética clínica desta população sofre uma série de alterações que interferem diretamente nos processos de absorção, distribuição, metabolização e eliminação dos medicamentos, podendo os medicamentos em doses terapêuticas habituais produzir efeitos tóxicos e reações medicamentosas adversas indesejáveis 8 .

Parte-se do princípio que a utilização de medicamentos é um processo social que deve estar sob o controle dos profissionais da saúde objetivando a diminuição de agravos à saúde. Entretanto, salienta-se a pressão mercadológica da indústria farmacêutica, seja diretamente sobre o consumidor, seja sobre os profissionais de saúde.

Neste contexto, o presente estudo farmacoepidemiológico avaliou o perfil do consumo de medicamentos por idosos residentes na zona urbana de Santa Rosa. O município situa-se na região noroeste do Estado do Rio Grande do Sul, Brasil, com população aproximada de 70 mil habitantes, sendo 6.323 (9\%) idosos acima de 60 anos, 2.691 homens $(42,6 \%)$ e 3.632 mulheres (57,6\%), a economia é baseada na agropecuária e na indústria metal-mecânica. Buscou-se analisar também a qualidade do uso de medicamentos nesta população, empregando indicadores capazes de identificar o padrão do uso, posologia, medicamento prescrito e não prescrito, forma de aquisição, prazo de validade e formas de armazenagem.

\section{Metodologia}

Foi realizado um estudo epidemiológico do tipo transversal com idosos na área urbana do Município de Santa Rosa, no ano de 2006, com o objetivo de caracterizar a utilização de medicamentos. A população em estudo incluiu pessoas com 60 anos ou mais, independente do gênero, que não estivessem hospitalizadas.

A seleção da amostra foi feita em estágios múltiplos, por conglomerados, utilizando os setores censitários do Instituto Brasileiro de Geografia e Estatística (IBGE). Através de sorteio aleatório simples foram sorteados 17 dos 72 setores censitários da cidade, nestes setores sortearam-se quarteirões de acordo com a população de idosos de cada setor e nestes quarteirões foram selecionados todos os idosos, garantindo-se, desta forma uma amostra equiprobabilística.

O tamanho de amostra foi calculado no Epi Info 6.04 (Centers for Disease Control and Prevention, Atlanta, Estados Unidos), utilizando-se os seguintes parâmetros estatísticos: população idosa de 5 mil pessoas, prevalência de uso de medicamentos de $80 \% 9$ margem de erro de 5,5 e nível de confiança de 95\%. Adicionou-se 30\% para controle de fatores de confusão e $10 \%$ para perdas, necessitando-se 271 indivíduos.

A população elegível, no trabalho de campo, foi de 309 idosos sendo que 11 recusaram-se a participar e quatro não foram encontrados em seus domicílios após três retornos.

As informações foram obtidas diretamente dos que eram capazes de se comunicar e com o cuidador ou parente responsável pela sua medicação, em caso de inconsciência do idoso.

A coleta dos dados foi realizada através de entrevista domiciliar, utilizando questionário padronizado, aplicados por três farmacêuticos, alunos do curso de pós-graduação lato sensu em Saúde Pública e alunos do sétimo semestre do curso de Farmácia da Universidade Regional do Noroeste do Estado do Rio Grande do Sul (UNIJUÍ). Realizou-se um estudo piloto com uma amostra de idosos residentes em setores não sorteados.

A prevalência do uso de medicamentos foi verificada através de uma questão fechada, na qual perguntava-se ao idoso: "O Senhor(a) utilizou algum medicamento na última semana?”. Caracterizou-se o uso de medicamento independente de prescrição médica. Excluiu-se da coleta de dados os chás, medicamentos fitoterápicos, medicamentos homeopáticos e florais.

Para proceder à identificação dos medicamentos utilizados, o entrevistador pedia que o idoso trouxesse ao seu alcance todos os medicamentos utilizados na semana anterior ao dia da 
entrevista. Assim, as informações eram obtidas diante da observação das caixas dos medicamentos, bulas, prescrições e informações obtidas do entrevistado. Analisou-se também a forma de armazenagem e data de validade dos medicamentos, considerou-se medicamento armazenado inadequadamente aqueles que se apresentaram fora de sua embalagem original, sem data de validade na caixa ou blister, misturados com outras especialidades farmacêuticas na mesma embalagem e embalagens que se apresentavam recortadas e/ou ilegíveis.

Os dados foram digitados e analisados no Epi Info 6.04. Foram realizadas duas digitações para diminuir possibilidade de erros, tornando os dados coletados mais fidedignos. Foi realizada análise descritiva, estratificada por sexo, das características da população e a relação destas variáveis com o uso de medicamentos. Os medicamentos foram classificados de acordo com seus subgrupos e classificação farmacológica.

O projeto de pesquisa seguiu as orientações da Resolução no. 196/96 e complementares do Conselho Nacional de Saúde, foi registrado no Sistema Nacional de Informação sobre Ética em
Pesquisa (SISNEP) e aprovado pelo Comitê de Ética em Pesquisa da UNIJUÍ. Os entrevistados ou familiares receberam termo de consentimento informado e a todos foi garantido o direito de não participar da pesquisa.

\section{Resultados}

Foram entrevistados 294 idosos com uma média de idade de 69,6 (DP = 7,6), $180(61,2 \%)$ eram mulheres, a maioria casada $(61,2 \%)$, mais da metade tinha até quatro anos de estudo, o rendimento do idoso era de até dois salários mínimos para quase dois terços da amostra. Observaram-se escolaridade e renda maiores entre os homens e o percentual de viuvez maior entre as mulheres (Tabela 1).

O coeficiente de prevalência do uso de medicamentos na última semana foi de $82 \%$, totalizando 827 medicamentos consumidos, distribuídos em 213 apresentações farmacêuticas diferentes. O número máximo de medicamentos utilizado por um idoso foi 13 , sendo que $92,2 \%$ tomavam até seis medicamentos (Tabela 2).

Tabela 1

Distribuição das variáveis sócio-demográficas de acordo com o gênero em idosos com mais de 60 anos. Santa Rosa, Rio Grande do Sul, Brasil, 2006.

\begin{tabular}{|c|c|c|c|c|c|}
\hline \multirow[t]{2}{*}{ Variável } & \multicolumn{2}{|c|}{ Mulheres } & \multicolumn{2}{|c|}{ Homens } & \multirow[t]{2}{*}{$p$} \\
\hline & $\mathbf{n}$ & $\%$ & $\mathbf{n}$ & $\%$ & \\
\hline \multicolumn{6}{|l|}{ Idade (anos) } \\
\hline $60-64$ & 58 & 32,2 & 35 & 30,7 & \\
\hline $65-69$ & 37 & 20,6 & 29 & 25,4 & 0,459 \\
\hline $70-74$ & 42 & 23,3 & 19 & 16,7 & \\
\hline 75 ou + & 43 & 23,9 & 31 & 27,2 & \\
\hline \multicolumn{6}{|l|}{ Escolaridade (anos de estudo) } \\
\hline 0 & 28 & 15,6 & 11 & 9,6 & \\
\hline $1-4$ & 79 & 43,9 & 46 & 40,4 & 0,126 \\
\hline $5-8$ & 56 & 31,1 & 37 & 32,5 & \\
\hline 9 ou + & 17 & 9,4 & 20 & 17,5 & \\
\hline \multicolumn{6}{|c|}{ Renda por idoso (salário mínimo) } \\
\hline Sem renda & 38 & 21,1 & 1 & 0,9 & \\
\hline$\leq 1$ & 85 & 47,2 & 51 & 44,7 & $<0,001$ \\
\hline $1,1-2$ & 34 & 18,9 & 25 & 21,9 & \\
\hline $2,1-3$ & 13 & 7,2 & 14 & 12,3 & \\
\hline 3,1 ou + & 10 & 5,6 & 23 & 20,2 & \\
\hline \multicolumn{6}{|l|}{ Estado civil } \\
\hline Casado(a) & 86 & 47,8 & 94 & 82,5 & \\
\hline Solteiro(a)/Divorciado(a) & 21 & 11,7 & 7 & 6,1 & $<0,001$ \\
\hline Viúvo(a) & 73 & 40,6 & 13 & 11,4 & \\
\hline Total & 180 & 100,0 & 114 & 100,0 & \\
\hline
\end{tabular}


Uso de medicamentos em idosos de acordo com gênero. Santa Rosa, Rio Grande do Sul, Brasil, 2006.

\begin{tabular}{|c|c|c|c|c|c|}
\hline \multirow[t]{2}{*}{ Variável } & \multicolumn{2}{|c|}{ Mulheres } & \multicolumn{2}{|c|}{ Homens } & \multirow[t]{2}{*}{$\mathrm{p}$} \\
\hline & $\mathbf{n}$ & $\%$ & $\mathrm{n}$ & $\%$ & \\
\hline \multicolumn{6}{|c|}{ Uso de medicamentos na última semana } \\
\hline $\operatorname{Sim}$ & 157 & 87,2 & 84 & 73,7 & 0,003 \\
\hline Não & 23 & 12,8 & 30 & 26,3 & \\
\hline \multicolumn{6}{|c|}{ Quanto ao modo de uso dos medicamentos } \\
\hline Prescritos & 529 & 96,2 & 265 & 95,7 & 0,721 \\
\hline Não-prescritos & 21 & 3,8 & 12 & 4,3 & \\
\hline \multicolumn{6}{|c|}{ Data de validade dos medicamentos } \\
\hline Não-vencidos & 541 & 98,5 & 275 & 99,3 & 0,509 \\
\hline Vencidos & 8 & 1,5 & 2 & 0,7 & \\
\hline \multicolumn{6}{|c|}{ Armazenamento dos medicamentos } \\
\hline Adequados & 537 & 97,8 & 274 & 98,9 & 0,262 \\
\hline Inadequados & 12 & 2,2 & 3 & 1,1 & \\
\hline \multicolumn{6}{|c|}{ Média de gasto mensal com compra de } \\
\hline medicamentos em reais & \multicolumn{2}{|c|}{$80,06(\mathrm{DP}=108,82)$} & \multicolumn{2}{|c|}{$92,63(\mathrm{DP}=121,26)$} & \\
\hline
\end{tabular}

A média de medicamentos utilizados por idoso foi de 2,79 (DP = 2,4). O gênero feminino apresentou uma média de 3,04 (DP = 2,4) e o gênero masculino 2,41 (DP = 2,4).

Dos 827 medicamentos utilizados pelos idosos, 794 (96\%) foram utilizados com prescrição médica. $\mathrm{O}$ uso de medicamentos não prescritos foi de $3,8 \%$ entre as mulheres e $4,3 \%$ entre os homens e a proporção de medicamentos vencidos foi de $1,5 \%$ e $0,7 \%$ respectivamente. Apenas 10 $(1,2 \%)$ dos medicamentos apresentaram data de validade expirada e 15 (1,8\%) apresentaram armazenamento inadequado (Tabela 2).

As classes farmacológicas mais utilizadas pela população idosa foram: anti-hipertensivos $(21,28 \%)$, diuréticos $(11,37 \%)$, medicamentos para circulação periférica $(6,53 \%)$, antiinflamatórios não-esteróides (5,68\%), antianginosos (5,68\%), hipnóticos e sedativos $(5,32 \%)$ e antiulcerosos $(5,08 \%)$ (Tabela 3$)$.

Os princípios ativos, dosagens e subgrupos farmacológicos mais freqüentemente utilizados foram: Captopril 25mg [inibidor da enzima conversora de angiotensina (9,5\%)]; Hidroclorotiazida $25 \mathrm{mg}$ [diurético tiazídico (6,8\%)]; Ácido Acetilsalicílico 100mg [inibidor não-seletivo da COX $(6,20 \%)]$; Omeprazol 20mg [inibidor da bomba de prótons $(4,40 \%)$ ]; Furosemida 40mg [diurético de alça $(2,8 \%)$ ]; Digoxina 0,25mg [glicosídeos cardíacos $(2,2 \%)]$ (Tabela 4$)$

\section{Discussão}

Realizou-se inquérito domiciliar em uma amostra representativa dos idosos do Município de Santa Rosa. As entrevistas realizadas por farmacêuticos com até três retornos aos domicílios e checagem sistemática das entrevistas asseguraram uma taxa consideravelmente pequena de perdas e garantiram a identificação precisa dos medicamentos utilizados.

As características sócio-demográficas da população em estudo refletiram em geral o quadro da população idosa no Brasil, havendo maior proporção de idosas 10,11. Dentre as hipóteses que explicam essa diferença destaca-se a que sustenta que os homens têm altas taxas de mortalidade precoce relacionadas à violência, acidentes de trânsito e doenças crônicas 12 .

Além disso, as mulheres possuem uma maior preocupação com a sua saúde e procuram mais os serviços do que os homens, principalmente em conseqüência aos vários programas de saúde (pré-natal, prevenção de câncer de colo uterino e de mama) direcionados especificamente ao gênero feminino ficando estas mais sujeitas a medicalização 13 .

A prevalência do uso de medicamentos na última semana estabelecida neste estudo foi de $82 \%$, sendo comparáveis ao encontrado por Texeira \& Lefreve 9 , $80 \%$ no ano de 2001, e Loyola Filho et al. 14, 79,1\% em um estudo de base populacional realizado em Bambuí, um município situado na região centro-oeste do Estado de Minas 
Classificação dos medicamentos utilizados por grupos farmacológicos. Santa Rosa, Rio Grande do Sul, Brasil, 2006.

\begin{tabular}{|c|c|c|}
\hline Principais grupos farmacológicos & $\mathbf{n}$ & $\%$ \\
\hline Analgésicos e antiinflamatórios & 92 & 11,12 \\
\hline Analgésicos não opióides & 30 & 3,63 \\
\hline Antiinflamatórios não-esteroidais & 47 & 5,68 \\
\hline Outros & 15 & 1,81 \\
\hline Antiinfecciosos & 11 & 1,33 \\
\hline Antibacterianos sistêmicos & 7 & 0,85 \\
\hline Antifúngicos sistêmicos e locais & 2 & 0,24 \\
\hline Outros & 2 & 0,24 \\
\hline Suplementos & 16 & 1,94 \\
\hline Antianêmicos & 8 & 0,97 \\
\hline Carbonato de cálcio + vitamina D & 7 & 0,84 \\
\hline Outros & 1 & 0,12 \\
\hline Sistema nervoso central & 106 & 12,82 \\
\hline Hipnóticos e sedativos & 44 & 5,32 \\
\hline Antidepressivos & 39 & 4,72 \\
\hline Outros & 23 & 2,78 \\
\hline Sistema cardiovascular & 452 & 54,65 \\
\hline Anti-hipertensivos & 176 & 21,28 \\
\hline Diuréticos & 94 & 11,37 \\
\hline Medicamentos para circulação periférica & 54 & 6,53 \\
\hline Antiarrítimicos & 22 & 2,66 \\
\hline Antilipêmicos & 20 & 2,42 \\
\hline Antianginosos & 47 & 5,68 \\
\hline Medicamentos para circulação cerebral & 27 & 3,26 \\
\hline Outros & 12 & 1,45 \\
\hline Sistema digestivo & 65 & 7,85 \\
\hline Antiulcerosos & 42 & 5,08 \\
\hline Antidiarréicos e antiespasmódicos & 1 & 0,12 \\
\hline Hepatoprotetores e digestivos & 2 & 0,24 \\
\hline Antiácidos & 4 & 0,48 \\
\hline Antieméticos & 3 & 0,36 \\
\hline Laxantes & 10 & 1,21 \\
\hline Outros & 3 & 0,36 \\
\hline Sistema respiratório & 33 & 4,00 \\
\hline Antiasmáticos & 24 & 2,90 \\
\hline Outros & 9 & 1,10 \\
\hline Sistema endócrino & 30 & 3,62 \\
\hline Insulina e outros antidiabéticos & 16 & 1,93 \\
\hline Hormônios da tireóide & 9 & 1,09 \\
\hline Outros & 5 & 0,60 \\
\hline Outros grupos farmacológicos & 22 & 2,66 \\
\hline Total de medicamentos utilizados na última semana & 827 & 100,00 \\
\hline
\end{tabular}

Gerais, no ano 1997, e em outro estudo realizado na região metropolitana de Belo Horizonte (2005) encontrou uma prevalência menor (72,1\%) 15.

A maioria dos estudos com idosos demonstrou que o número médio de medicamentos utilizado por pessoa fica entre 3 a 3,69 9,13,16. No entanto, em estudo realizado em Belo Horizonte, observou-se uma média de 2,18, sugerindo que em regiões distintas do país existem diferenças no consumo de medicamentos. A média encontrada nesta pesquisa foi 2,79 $(\mathrm{DP}=2,4)$ medicamentos por idoso, podendo ainda ser considerada elevada. $\mathrm{O}$ acesso fácil a medicações e a falta de conscientização do uso de medidas não far- 
Princípios ativos e subgrupos mais freqüentes utilizados pelos idosos na semana anterior à entrevista. Santa Rosa, Rio Grande do Sul, Brasil, 2006.

\begin{tabular}{|c|c|c|c|}
\hline Princípio ativo e dosagens & Subgrupos & $\mathbf{n}$ & $\%$ \\
\hline Captopril 25mg & Inibidor da enzima conversora de angiotensina & 77 & 9,50 \\
\hline Hidroclorotiazida $25 \mathrm{mg}$ & Diurético tiazídico & 55 & 6,80 \\
\hline Ácido acetilsalicílico 100mg & Inibidor não-seletivo da COX & 50 & 6,20 \\
\hline Omeprazol 20mg & Inibidor da bomba de prótons & 36 & 4,40 \\
\hline Furosemida 40mg & Diurético de alça & 23 & 2,80 \\
\hline Digoxina 0,25 mg & Glicosídeos cardíacos & 18 & 2,20 \\
\hline Amiodarona 200mg & Bloqueadores de canais de potássio & 17 & 2,10 \\
\hline Mononitrato de isossorbida $20 \mathrm{mg}$ & Nitrato & 17 & 2,10 \\
\hline Propatilnitrato $10 \mathrm{mg}$ & Nitrato & 15 & 1,80 \\
\hline Propranolol 40mg & Beta-bloqueador não cardiosseletivo & 14 & 1,70 \\
\hline Diclofenaco sódico 50mg & Antiinflamatórios não-esteroidais & 14 & 1,70 \\
\hline Diclofenaco potássico $50 \mathrm{mg}$ & Antiinflamatórios não-esteroidais & 14 & 1,70 \\
\hline Cloridrato de fluoxetina $20 \mathrm{mg}$ & Inibidores seletivos da recaptação de serotonina & 13 & 1,60 \\
\hline Hidroclorotiazida 50mg & Diurético tiazídico & 12 & 1,50 \\
\hline Metildopa 500mg & Inibidor do sistema simpático central & 12 & 1,50 \\
\hline Cinarizina $75 \mathrm{mg}$ & Bloqueador dos receptores $\mathrm{H} 1$ & 11 & 1,40 \\
\hline Diazepan 10mg & Benzodiazepínico & 10 & 1,20 \\
\hline Flunarizina $10 \mathrm{mg}$ & Bloqueador dos receptores $\mathrm{H} 1$ & 10 & 1,20 \\
\hline
\end{tabular}

macológicas, podem estar contribuindo para o consumo de medicamentos nesta população. O elevado percentual de consumo confirma a importância dos medicamentos na atenção à saúde do idoso.

Loyola Filho et al. 15 destacam que os tipos de medicamentos consumidos e os fatores associados ao consumo são muito semelhantes entre as populações, sugerindo que existe certa uniformidade no tipo de medicamento e nos seus determinantes. Por este motivo, é importante a realização de estudos nas mais diversas regiões para estabelecer o consumo de medicamentos na sua própria população e subsidiar os gestores públicos na programação de serviços e assistência farmacêutica para idosos.

As classes terapêuticas mais utilizadas por idosos foram similares ao descrito na literatura $15,16,17$. Os medicamentos para o sistema cardiovascular são os mais freqüentemente prescritos pelos médicos, pois as doenças cardiovasculares lideram as causa de morbi-mortalidade em indivíduos acima de 65 anos 15,17. Observou-se também que os medicamentos para o sistema nervoso central, analgésicos e antiinflamatórios são amplamente utilizados revelando o desconforto que o "estar ansioso" e as dores agudas causam nos idosos. Cabe ressaltar que o uso concomitante destas classes terapêuticas pode levar a interações medicamentosas perigosas 6 .
Os resultados deste estudo onde mais de $30 \%$ dos entrevistados estava utilizando diariamente pelo menos três medicamentos diferentes parecem confirmar que o uso concomitante de diversas especialidades farmacêuticas é uma prática comum entre os idosos em todo o mundo. Semelhante ao encontrado por Almeida et al. $18 \mathrm{em}$ idosos atendidos em um serviço ambulatorial de saúde mental na cidade de São Paulo, Sharkey et al. 19 em um estudo com 326 idosos realizado no Texas, Estados Unidos, evidenciaram que 31,6\% dos entrevistados utilizava cinco ou mais classes terapêuticas, e Pizzuti et al. 20, na Itália, demonstraram que $45,3 \%$ utilizavam quatro ou mais medicamentos diferentes por dia.

Segundo Mosegui 6, os idosos chegam a constituir 50\% dos multiusuários de medicamentos, sendo comum encontrar em suas prescrições uso de fármacos de uma mesma classe terapêutica, sem valor terapêutico.

Observou-se também que o uso de medicamentos para o sistema respiratório e antiinfecciosos foi pouco freqüente, uma possível explicação seria a época de realização da pesquisa, janeiro a junho de 2006, não contemplando o inverno, clima que aumenta a incidência de patologias respiratórias.

A porcentagem de medicamentos utilizados sem prescrição médica foi baixa, de forma similar ao encontrado por Loyola Filho et al. 14, 
mas diferente do demonstrado por Veras 11, onde quase $30 \%$ dos idosos consumiam medicamentos sem prescrição. Esse fato pode ser explicado, pois os medicamentos considerados de uso contínuo, muito usados nesta faixa etária, na maioria das vezes são prescritos pelos médicos. No caso de Santa Rosa, o SUS utiliza a Estratégia de Saúde da Família há mais de dez anos, dispondo de uma rede de atenção integral composta por 13 equipes propiciando cobertura de quase $100 \%$ e fornecendo gratuitamente a maior parte dos medicamentos utilizados pelos doentes crônicos. Reforçando esta hipótese, verificamos que os medicamentos e forma de apresentação mais freqüentemente relatados foram os existentes na lista básica dispensados gratuitamente na rede básica de saúde do município.

Outra explicação poderia ser o viés de memória, ou seja, os medicamentos de uso contínuo não seriam esquecidos pelos idosos, por estarem incorporados a sua rotina diária, diferentemente dos medicamentos de uso esporádico, geralmente comprados sem prescrição médica. Segundo Texeira \& Lefreve 9 (p. 210), "a falha para relembrar os medicamentos tomados é um fato comum na população geral de idosos".

Conforme Coelho et al. 17, torna-se difícil comparar estudos relacionando o uso de medicamentos prescritos e não-prescritos, pois se pode confundir o termo medicamento não-prescrito com os medicamentos não tarjados ou de venda livre dispensados tanto na unidade básica como em farmácias e drogarias.

Nosso estudo confirmou que os homens idosos tomam mais medicamentos não-prescritos do que as mulheres, como já demonstrado por Loyola Filho et al. 15 em um estudo de base populacional em idosos realizado na metropolitana de Belo Horizonte. Neste sentido, observamos também que os homens gastam mais com aquisição de medicamentos, provavelmente em função de comprar medicamentos não prescritos diretamente nas farmácias e drogarias.

No entanto, os gastos com medicamentos podem ter sido subestimados neste estudo em função da desconfiança dos idosos em responder as questões relacionadas com a compra e uso de medicamentos, temendo uma vinculação com o fornecimento gratuito nas unidades básicas de saúde.

O estudo conseguiu demonstrar o elevado número de idosos que utilizam medicamentos, as principais classes farmacológicas e apresentações utilizadas, bem como algumas características associadas com o uso de medicamentos não prescritos. Contudo, novos estudos são necessários para permitir uma compreensão de toda a complexidade do tema. Estudos como estes são importantes, pois podem ser utilizados como ferramenta para reorientação da assistência farmacêutica.

Tornar a terapia medicamentosa da população idosa eficiente e eficaz é tarefa de todos os profissionais da saúde: médicos, farmacêuticos, enfermeiros e outros, que devem instituir educação permanente tanto em relação aos avanços tecnológicos na área farmacêutica, como no comportamento desta população em relação ao uso e armazenamento correto dos medicamentos.

Assim, o processo de acompanhamento farmacoterapêutico do paciente idoso é fundamental para a promoção do uso racional de medicamentos, para contribuir no processo educativo dos usuários acerca da automedicação , da interrupção e da troca do tratamento prescrito, bem como a necessidade da receita médica; realizando uma avaliação dos medicamentos empregados pelos idosos quanto à complexidade de regime posológico, custo e aderência ao tratamento.

\section{Resumo}

Os medicamentos representam um dos itens mais importantes da atenção à saúde do idoso. Estes particularmente utilizam múltiplos medicamentos apresentando mais reações adversas. O estudo objetivou avaliar o perfil do consumo de medicamentos por idosos. Desenvolveu-se um estudo epidemiológico transversal mediante entrevistas domiciliares, numa amostra aleatória de 294 idosos, residentes em Santa Rosa, Rio Grande do Sul, Brasil. Os idosos representam 9\% da população. Os resultados evidenciaram consumo médio de 2,79 medicamentos por idoso. O coeficiente de prevalência do uso de medicamentos na última semana foi de 82\%, dos 827 medicamentos utilizados pelos idosos 794 (96\%), foram prescritos. As classes farma- cológicas mais utilizadas foram os anti-hipertensivos (21,3\%) e diuréticos (11,3\%). A prevalência do uso de medicamentos foi comparável ao encontrado em outros estudos, confirmando a importância dos medicamentos na atenção à saúde do idoso. A porcentagem de medicamentos utilizados sem prescrição médica foi baixa. Os medicamentos e forma de apresentação mais freqüentemente relatados foram os usualmente prescritos e dispensados gratuitamente na rede básica de saúde do município que está estruturada há mais de 10 anos com quase $100 \%$ de cobertura.

Preparações Farmacêuticas; Idoso; Farmacoepidemiologia 


\section{Colaboradores}

V. B. Flores e L. A. Benvegnú realizaram a análise e discussão dos dados e a redação do artigo. V. B. Flores coordenou a aplicação dos questionários, supervisionou o trabalho de campo e realizou a codificação dos dados.

\section{Agradecimentos}

Este trabalho contou com o apoio da Fundação Municipal da Saúde de Santa Rosa e da UNIJUÍ; e recebeu recurso financeiro para sua execução do Conselho Nacional de Desenvolvimento Científico e Tecnológico (CNPq, aprovado pelo protocolo no. 402808/2005-3). Agradecemos também a todos os idosos que participaram deste estudo e aos entrevistadores: alunos do sétimo semestre de 2006 do curso de Farmácia e do curso de pós-graduação lato sensu em Saúde Pública da UNIJUÍ.

\section{Referências}

1. Andrade MA, Silva VS, Freitas O. Assistência farmacêutica como estratégia para o uso racional de medicamentos em idosos. Semina Ciênc Biol Saúde 2004; 25:55-63.

2. Karnikowski MGO, Nóbrega OT, Naves JOS, Silver LD. Access to essential drugs in 11 Brazilian cities: a community based evaluation and action method. J Public Health Policy 2004; 25:288-98.

3. Haak H. Padrões de consumo de medicamentos em dois povoados da Bahia. Rev Saúde Pública 1989; 23:143-51.

4. Barros MBA. Saúde e classe social: um estudo sobre morbidade e consumo de medicamentos [Tese de Doutorado]. São Paulo: Faculdade de Medicina de Ribeirão Preto, Universidade de São Paulo; 1983.

5. Franco RCS, Carvalho NJA, Khouri MA, Nunes MO, Santos JJW, Marques NJ. Consumo de medicamentos em um grupo populacional da área urbana de Salvador - BA. Rev Baiana Saúde Pública 1987; 13/14:113-21.

6. Mosegui GB, Rozenfeld S, Veras RP. Vianna CM. Avaliação da qualidade do uso de medicamentos em idosos. Rev Saúde Pública 1999; 33:437-44.

7. Veras RP. Em busca de uma assistência adequada à saúde do idoso: revisão da literatura e aplicação de um instrumento de detecção precoce e de previsibilidade de agravos. Cad Saúde Pública 2003; 19:705-15.

8. Katzung BG. Aspectos especiais da farmacologia geriátrica. In: Katzung BG, organizador. Farmacologia básica \& clínica. 8a Ed. Rio de Janeiro: Editora Guanabara-Koogan; 2002. p. 889-906.

9. Teixeira JJV, Lefevre F. A prescrição medicamentosa sob a ótica do paciente idoso. Rev Saúde Pública 2001; 35:207-13.

10. Coelho JM, Ramos LR. Epidemiologia do envelhecimento no Noroeste do Brasil: resultados de inquérito domiciliar. Rev Saúde Pública 1999; 33:445-53.
11. Veras RP. País jovem com cabelos brancos: a saúde do idoso no Brasil. Rio de Janeiro: Relume-Dumará/Universidade do Estado do Rio de Janeiro; 1994

12. Veras RP. Modelos contemporâneos no cuidado à saúde: novos desafios em decorrência da mudança do perfil epidemiológico da população brasileira. Revista USP 2001; 51:72-85.

13. Bertoldi AD, Barros AJD, Hallal PC, Lima RC. Utilização de medicamentos em adultos: prevalência e determinantes individuais. Rev Saúde Pública 2004; 38:228-38.

14. Loyola Filho AI, Uchoa E, Firmo JOA, Lima-Costa MF. Estudo de base populacional sobre o consumo de medicamentos entre idosos: Projeto Bambuí. Cad Saúde Pública 2005; 21:545-53.

15. Loyola Filho AI, Uchoa E, Lima-Costa MF. Estudo epidemiológico de base populacional sobre uso de medicamentos entre idosos na Região Metropolitana de Belo Horizonte, Minas Gerais, Brasil. Cad Saúde Pública 2006; 22:2657-67.

16. Flores LM, Mengue SS. Uso de medicamentos por idosos em região do sul do Brasil. Rev Saúde Pública 2005; 39:924-9.

17. Coelho JM, Marcopito LF, Castelo A. Perfil de utilização de medicamentos por idosos em área urbana do Noroeste do Brasil. Rev Saúde Pública 2004; 38:557-64

18. Almeida OP, Ratto L, Garrido R, Tamal S. Fatores preditores e conseqüências clínicas do uso de múltiplas medicações entre idosos atendidos em um serviço ambulatorial de saúde mental. Rev Bras Psiquiatr 1999; 21:3-19.

19. Sharkey JR, Browne B, Ory MG, Wang S. Patterns of therapeutic medication category use among community-dwelling homebound older adults. Pharmacoepidemiol Drug Saf 2005; 14:715-23.

20. Pizzuti R, Caffari B, Binkin N; Gruppo ARGENTO. La prescrizione di farmaci nell'anziano: risultati dello studio Argento. Ig Sanita Pubbl 2006; 62:11-26.

Recebido em 29/Mar/2007

Versão final reapresentada em 24/Ago/2007

Aprovado em 26/Set/2007 